\title{
Low/Adaptive Precision Computation in Preconditioned Iterative Solvers for III-Conditioned Problems
}

\author{
Masatoshi Kawai \\ masatoshi.kawai@riken.jp \\ The University of Tokyo, Information Technology Center \\ Tokyo, Japan
}

\begin{abstract}
Double precision(FP64) is generally used in computer science, and an incomplete Cholesky preconditioned conjugate gradient(ICCG) method, which is widely used in computer simulations, is a typical example. Recently, the use of single-precision(FP32) in the ICCG method with well-conditioned problems is discussed for reducing computational time and power consumption. Also, the use of halfprecision(FP16) is examined in the field of machine learning, and hardware support of FP16 on GPUs and some CPUs is advancing. Using FP16 can further reduce the amount of memory translation from FP32, so high effects can be expected if it can be used with the ICCG method. When we apply FP16 to the ICCG method, It is difficult to solve the ill-condition problem because of the poor expressiveness of the exponent of FP16. Therefore, in this study, we evaluate the usefulness of FP16 in the ICCG method with several conditions of the problem, and we also proposed the implementation for CPU of and evaluated FP21 and FP42 adaptive precision whose expressiveness is higher than FP16. Additionally, to reduce the calculation time by using low precision, it is necessary to maintain high memory width consumption by an effective SIMDization. Therefore, in this study, we also evaluate the performance of ELLPACK(ELL) and Sell-C- $\sigma$ storage formats, which can efficiently use SIMDization.
\end{abstract}

\section{CCS CONCEPTS}

- Mathematics of computing $\rightarrow$ Mathematical software performance; Solvers.

\section{KEYWORDS}

Low-precision, Adaptive-precision, ICCG, Sell-C- $\sigma$

\section{ACM Reference Format:}

Masatoshi Kawai and Kengo Nakajima. 2022. Low/Adaptive Precision Computation in Preconditioned Iterative Solvers for Ill-Conditioned Problems. In International Conference on High Performance Computing in Asia-Pacific Region (HPC Asia2022), fanuary 12-14, 2022, Virtual Event, fapan. ACM, New York, NY, USA, 11 pages. https://doi.org/10.1145/3492805.3492813

Permission to make digital or hard copies of all or part of this work for personal or classroom use is granted without fee provided that copies are not made or distributed for profit or commercial advantage and that copies bear this notice and the full citation on the first page. Copyrights for components of this work owned by others than ACM must be honored. Abstracting with credit is permitted. To copy otherwise, or republish, to post on servers or to redistribute to lists, requires prior specific permission and/or a fee. Request permissions from permissions@acm.org.

HPC Asia2022, January 12-14, 2022, Virtual Event, Japan

(c) 2022 Association for Computing Machinery.

ACM ISBN 978-1-4503-8498-8/22/01 ..\$15.00

https://doi.org/10.1145/3492805.3492813

\author{
Kengo Nakajima \\ The University of Tokyo, Information Technology Center \\ Tokyo, Japan \\ RIKEN Center for Computational Science \\ Kobe, Japan
}

\section{INTRODUCTION}

In recent computer science research, the use of low-precision floatingpoint formats such as single-precision(FP32) and half-precision(FP16) was reexamined, and mixed-precision was also studied to reduce computational time and power consumption. The use of lower precision formats such as FP32, FP16, and FP8 has been widely discussed and has yielded practical applications[6,11]. The recent GPU and A64FX CPU of Fugaku and Wisteria/BDEC-01 Odyssey supercomputer support FP16. In response to such situations, the use of FP32 in computer simulations has also been reexamined, and it has been reported that the accuracy of FP32 is practical[5, 7, 12]. However, there are few practical examples of FP16[14] because of poor expressive ability.

In the research of low-precision formats, an adaptive precision such as FP21, which is not standardized by IEEE754, was proposed. The expressive ability of the exponential part of FP16 is little. The digit number of the exponential part is 3 , and it is often unusable for practical use. In the research by Yamaguchi et al.[20], FP21 precision formats were proposed, and FP21 contributed to accelerating seismic simulation on GPUs. FP21 has the same expressive ability of exponential part as FP32 and is more suitable for computer simulations than FP16.

An incomplete Cholesky(IC) preconditioner for a conjugate gra$\operatorname{dient}(\mathrm{CG})$ method is one of the applications expected to reduce computational time by applying low-precision formats. The IC preconditioned CG(ICCG) method, widely used as a solver for implicit methods in numerical simulations, is a typical example of how FP64 is generally used. This is because the convergence of the CG method is strongly affected by the calculation accuracy. Because the performance of the ICCG method generally depends on the memory bandwidth, we can expect that applying low-precision formats reduces the computational time of the ICCG method. In particular, the IC preconditioner has a certain effect with low accuracy because the target equation can be simply solved by the preconditioner[16], and the IC preconditioner takes approximately half of the computational time of the entire ICCG method.

In this study, we applied low-precision floating-point formats such as FP32, FP21, and FP16 to the IC preprocessing of a steadystate 3D heat conduction(P3D) application[17] and evaluated its effects on the convergence rate and reduced computational time. In addition, we also proposed and evaluated FP42, which has an expressive ability between FP32 and FP64. Because FP21 has only been evaluated on GPUs so far[20], this research proposes an optimized implementation for CPUs, including FP42. In this evaluation, we apply low-precision formats to the matrix and vectors referenced in the IC preconditioner. To ensure sufficient access to memory 


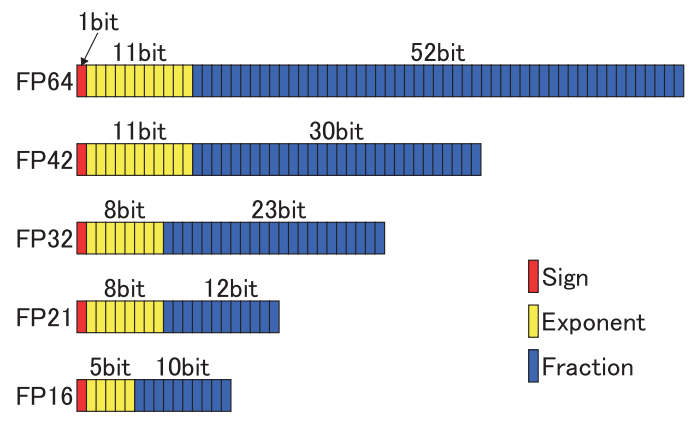

Figure 1: Bits layout of FP64, FP42, FP32, FP21 and FP16

even with low-precision formats, the sparse matrix storage format in this application is not limited to compress row storage(CRS), but also ELLPACK(ELL)[3] and Sell-C- $\sigma[9]$. The effect on convergence rate was evaluated by assuming a set of coefficient matrices from a small condition number to a large condition number. Then, the matrices with small and large condition number are expressed as well- and ill-conditioned, respectively. In the evaluation, we used one node of Oakfores-PACS, Oakbridge-CX, or Wisteria/BDEC01 Odyssey supercomputers, respectively.

\section{FLOATING-POINT FORMATS}

In this section, we introduce the floating-point formats used in this study. The FP21 and FP42 formats are not supported by the general FPU. We also introduce how to handling these formats.

\subsection{Expressive ability of each floating-point}

This section describes the expressive ability of each floating-point format used in this study.

Modern compilers handle several formats. The quad(FP128), double(FP64), single(FP32), and half (FP16) formats are standardized by IEEE754[2]. Each format has a sign, exponential, and fraction part, and the widths of the exponent and fraction fields are different for each. Figure 1 shows the bit layout for each format. the higher the precision floating-point format, the wider the exponent and fraction fields become

FP21 has been proposed to reduce memory transfer in applications where FP16 has insufficient calculation accuracy. The width exponent field of FP21 is the same as that of FP32, but the fraction field is shortened from 23 to 12 bits (Figure 1). By changing floatingpoint formats from FP32 to FP21, the amount of data transfer is

Table 1: Expressive ability of each formats in decimal

\begin{tabular}{|c|r|r|}
\hline Formats & $\begin{array}{c}\text { Significand : } \\
\text { Number of decimal digits }\end{array}$ & $\begin{array}{c}\text { Exponent : Maximum } \\
\text { exponent in decimal }\end{array}$ \\
\hline FP64 & 15.95 & 308 \\
\hline FP42 & 9.33 & 308 \\
\hline FP32 & 7.22 & 38 \\
\hline FP21 & 3.91 & 38 \\
\hline FP16 & 3.31 & 5 \\
\hline
\end{tabular}

reduced to approximately $2 / 3$, so it can be expected to reduce the calculation time in applications regulated by memory bandwidth.

In this study, we also propose an FP42 format. The fraction of FP42 was reduced from 52 to 30 bits of FP64. Similar to FP21, we can expect to reduce the amount of data transfer to $2 / 3$ with an application for which FP64 is sufficient but FP32 is insufficient.

Table 1 shows the number of decimal digits that can be expressed in a significand and decimal maximum exponential value in the exponent. In Table 1, the number of decimal digits is calculated as follows:

$$
\begin{aligned}
10^{y} & =2^{(x+1)} \\
y & =(x+1) \log _{10} 2
\end{aligned}
$$

Subsequently, $y$ denotes the number of decimal digits, and $x$ denotes the width of the fraction field. The significand figure of floatingpoint formats includes a hidden bit. Then, the significand is denoted as $x+1$. When the width of the fraction is halved, the number of decimal digits is halved. In contrast, when the width of the exponent is halved, the maximum exponent value in decimal becomes a tenth. The maximum exponent value in the decimal of FP16 is too short, and it sometimes causes calculation failure in iterative methods.

\subsection{Load and store of FP21 and FP42}

This section describes how to handle FP21 and FP42 for computation. Furthermore, we use Fortran language for this study because the target application is written in Fortran.

FP21 and FP42 are classified into adaptive precision formats, which are not supported by compilers and general FPUs. FP21 and

\section{Pseudo-code 1: Typecasting from FP32 to FP21}

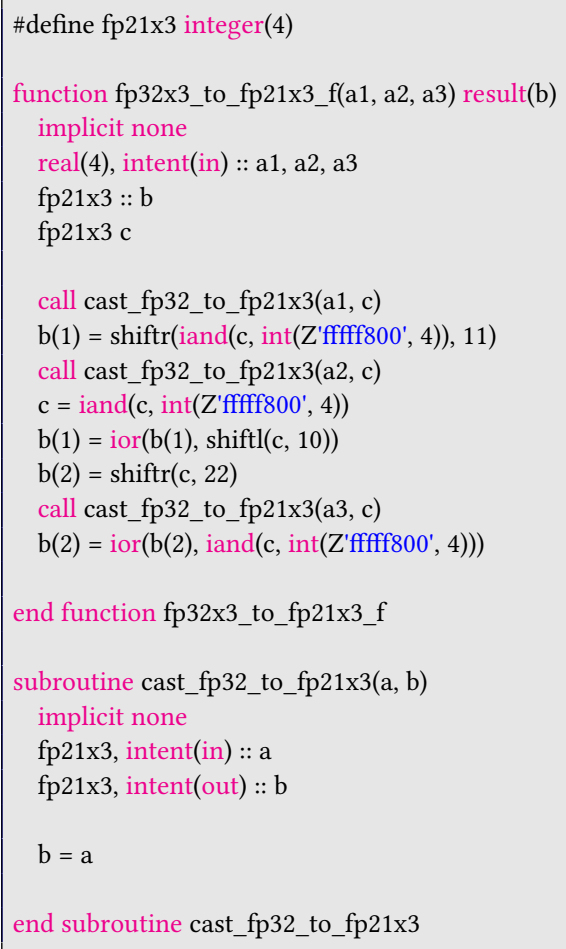




\section{Pseudo-code 2: Typecasting from FP21 to FP32}

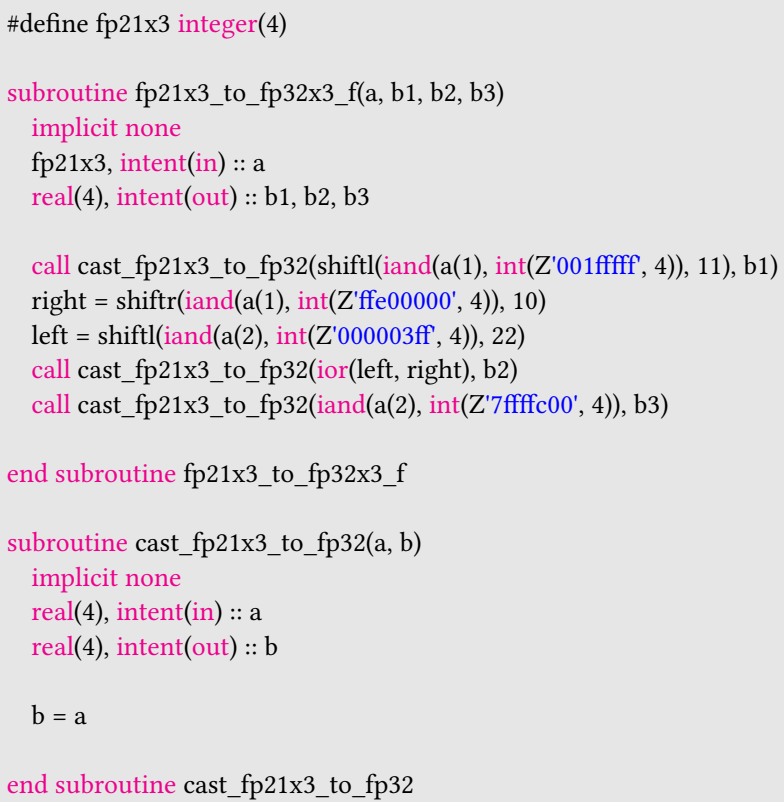

FP42 are cast to standard data types to compute them and then standard data types are cast to FP21 and FP42 for storage. In this study, we propose to cast three FP21 data and three FP42 data into two 32-bit integer data types and two 64-bit integer data types, respectively. For computing, we cast FP21 to FP32 and FP42 to FP64. $\mathrm{Alg}(1)$ and $\mathrm{Alg}(2)$ show a Fortran pseudo-code of typecasting routines between FP21 and FP32, and the typecasting processes from FP32 to FP21 are as follows:

(1) Typecasting from FP32(real(4)) to fp21x3(integer(4)) by using a function "cast_fp32_to_fp21x3" (Just changing handling data type in program)

(2) Extracting 21-bits values from the converted data using "AND" operation with the bitmask

(3) Applying a logical shift (not an arithmetic shift) by using shiftr or shiftl(Standardized by Fortran 2008)

(4) Substituting shifted value to target FP21x3 with "AND" operation (Required AND operation to keep substituted the other value)

The typecasting from FP21 to FP32 reverses the previous processes. The typecasts between FP42 and FP64 are similar. The differentials are mask bits and the width of the logical shifting. In the typecast, we use the "cast_fp32_tp_fp21x3" function. This function simply changes the data type from FP21 to a 32-bit integer without changing the internal bit information. In general typecasts, the internal bit information is updated such that the mathematical information does not change. In the typecast between FP21 and FP32, such a "cast_fp32_tp_fp21x3" function is required because it is necessary to change the handling data type in the language. This can be achieved by exchanging pointers in $C$ language, but the same approach cannot be made in Fortran. Then, the "cast_fp32_to_fp21x3" function receives real(4) data type as an integer(4) implicitly to convert in our Fortran implementation.

In previous research on FP21 by Yamaguchi et al.[20], three FP21 values were stored to one 64-bit integer data type. In our implementation, we use two 32 bit integer data types to match the width of the stored and computation data types. To consider an efficient SIMDization for the CPU, the width of all data types handled in the SIMDized loop should be the same. The widest data type regularizes the number of computing data per SIMD instruction. For example, when the CPU support 512bits SIMD instructions and a SIMDized loop includes one 64 bit and several 32 bit data types, the number of computations per SIMD instruction becomes eight because it is regularized by a 64 bit data type.

The typecasting routines are called every computation and it is an inside of the SIMDized loops. Therefore, a low overhead and SIMDizable are important. Then, we focus on inline expansion. If the typecasting routine is inlaid when compiling the program, all routines become SIMDizable, and latencies calling routines are minimized by software pipelining and working of an out-of-order engine. In the specification of the Fortran language, there are no directives or instructions to request inline expansion. However, inline expansion is a general optimization approach, and a compiler can be expected to inline the typecasting functions using a linktime optimizer. The authors confirmed that the typecasting routines are inlaid on the GNU compiler version 9.3.1, with "-O3 -flto" options and on Intel compiler version 19.1.3.304 with "-O3 -ipo" options. To confirm inline expansion, we checked the optimization reports of each compiler that is output with a compiler option "-foptinfo-inline" on GNU and "-qopt-report" on Intel. With the Fujitsu compiler, we cannot confirm the expected inline expansion because of the limitation that external procedures whose definition of argument and passed do not match ("cast_fp32_tp_fp21x3") do not perform inline expansion. Therefore, the numerical experiments did not include the evaluation of FP21 and FP42 by the Fujitsu compiler.

\section{RELATED RESEARCHES}

Computational approaches using low or multiple floating-point formats have been widely studied in the field of deep learning $[8,13]$. It has also been studied in several computational simulations, and there are several reports[4, 10]. Against this background, research applying low precision to iterative methods has been conducted in recent years[14, 15, 17, 18].

Focusing on the adaptive precision formats, there are studies on adaptive precisions such as the bfloat of an AI library by Gooble[1]. FP21 is proposed for seismic simulation on GPUs [20], which is applied to AXPY, dot-product, and matrix-vector multiplication. By using FP21, the performance of the seismic simulation improved by approximately $10 \%$.

In response to these previous studies, this study proposes and evaluates the effective implementation of FP21 on a CPU. Proposed FP21 in the paper Yamaguchi et al., FP21 is stored as a 64-bit integer data type. We propose storing FP21 to two 32-bit integer data types for effective SIMDization. We also describe the implementation that encourages the compiler to perform inline expansion. In addition, we also propose the FP42 data type. In this study, we also focused 
on evaluating versatility by using the problem that can control the conditions of the coefficient matrices by changing a parameter.

\section{P3D APPLICATION}

This section describe the properties of coefficient matrices and solver of the P3D application.

\subsection{Property of coefficient matrix}

In this section, we introduce how to control the condition of problems derived from the P3D application.

In the P3D application, a finite volume method was used to discretize following Poisson's equation.

$$
\nabla \cdot(\lambda \nabla \phi)=\rho, \quad \phi=0 \text { at } z=z_{\max }
$$

Then, $\phi$ denotes the thermal distribution, and $\rho$ denotes the given heating energy. The parameter $\lambda$ denotes the thermal diffusivity, and it has heterogeneity in the target object. The thermal diffusivity was calculated using the density, specific heat capacity, and thermal conductivity of the target material. The maximum and minimum values of thermal diffusivity are not clear, but in this study, we consider its as $10^{10}$.

The target problem is discretized on a structure grid with $n x \times$ $n y \times n z$ grids. Then, we denote a vector of $\phi$ and $\rho$ on the discretized space as $\boldsymbol{x}, \boldsymbol{b}$, an element $a_{i, j}$ of a coefficient matrix $\boldsymbol{A}$ of the system of linear equation(SLE) $\boldsymbol{A x}=\boldsymbol{b}$ to be solved is calculated as follows:

$$
a_{i, j}= \begin{cases}-\frac{d y \cdot d z}{\frac{d x}{2}\left(\frac{1}{\lambda_{x, y, z}}+\frac{1}{\lambda_{x-1, y, z}}\right)}, & j=i-1 \\ -\frac{d y \cdot d z}{\frac{d x}{2}\left(\frac{1}{\lambda_{x, y, z}}+\frac{1}{\lambda_{x+1, y, z}}\right)}, & j=i+1 \\ -\frac{d x \cdot d z}{\frac{d y}{2}\left(\frac{1}{\lambda_{x, y, z}}+\frac{1}{\lambda_{x, y-1, z}}\right)}, & j=i-n x \\ -\frac{d x \cdot d z}{\frac{d y}{2}\left(\frac{1}{\lambda_{x, y, z}}+\frac{1}{\lambda_{x, y+1, z}}\right)}, & j=i+n y \\ -\frac{d x \cdot d y}{\frac{d z}{2}\left(\frac{1}{\lambda_{x, y, z}}+\frac{1}{\lambda_{x, y, z-1}}\right)}, & j=i-n x \times n y \\ -\frac{d x \cdot d y}{\frac{d z}{2}\left(\frac{1}{\lambda_{x, y, z}}+\frac{1}{\lambda_{x, y, z+1}}\right)}, & j=i+n x \times n y \\ \sum_{k=1}^{N}-a_{i, k}, & j=i \\ 0, & \text { others }\end{cases}
$$

Then, $d x, d y$, and $d z$ denote the distance between the grids in the $x, y$, and $z$ directions, respectively. As mentioned above, we assume $\lambda$ to be $1.0 \leq \lambda \leq 10^{10}$, and the ratio of the maximum and minimum non-zero elements is on the order of 10 billion. The condition number of the coefficient matrix $A$ deteriorates as the ratio increases.

\subsection{ICCG method}

Figure 2 shows the computational process of the ICCG method. In this figure, $k$ denotes the number of iterations, $\boldsymbol{r}^{k}$ denotes residual vector, $\boldsymbol{p}^{k}$ denotes independent vector, and $\boldsymbol{q}$ denotes a storing vector for a result of preconditioning. The matrix $\dot{L}, \dot{D}$ and $\dot{U}$ denotes a strictly upper triangular, diagonal and narrowly lower triangular matrix which is derived from the incomplete Cholesky factorization.
"Red" : Matrices stored low precision

"Blue" : Vectors stored low precision

do $k=1, \quad$ !until converge

$$
\begin{aligned}
& \alpha=\frac{\left(\boldsymbol{r}^{k}, \boldsymbol{p}^{k}\right)}{\left(\boldsymbol{p}^{k}, \boldsymbol{A} \boldsymbol{p}^{k}\right)} \\
& \boldsymbol{x}^{k+1}=\boldsymbol{x}^{k}+\alpha \boldsymbol{p}^{k} \\
& \boldsymbol{r}^{k+1}=\boldsymbol{r}^{k}-\alpha \boldsymbol{A} \boldsymbol{p}^{k} \\
& \dot{\boldsymbol{r}}=\boldsymbol{r}^{k+1} \\
& \boldsymbol{q}=\dot{U}^{-1} \dot{\boldsymbol{D}}^{-1} \dot{L}^{-1} \dot{\boldsymbol{r}} \quad \text { !IC preconditioning } \\
& \beta=-\frac{\left(\boldsymbol{q}, \boldsymbol{A} \boldsymbol{p}^{k}\right)}{\left(\boldsymbol{p}^{k}, \boldsymbol{A} \boldsymbol{p}^{k}\right)} \\
& \boldsymbol{p}^{k+1}=\boldsymbol{q}+\beta \boldsymbol{p}^{k}
\end{aligned}
$$

enddo

Figure 2: Computational process of the ICCG method

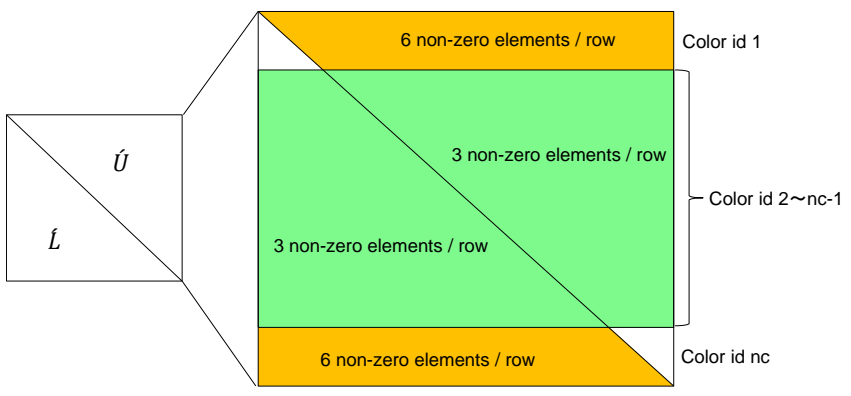

Figure 3: Mapping of non-zero elements after multi-coloring

In this study, we apply a low-precision formats to the matrix $\dot{L}$, $\dot{D}, \dot{U}$, and vector $\dot{\boldsymbol{r}}, \boldsymbol{q}$. If we apply the low-precision floating-point to the coefficient matrix $A$ used in the iteration of the CG method, the problem to be solved itself may change depending on the condition of the coefficient matrix. If we apply the low-precision floating point to the independent vector $\boldsymbol{p}$ or residual vector $\boldsymbol{r}$, it significantly decreases the convergence ratio owing to the deterioration of the accuracy of the inner product. Applying a low-precision floating point to the IC preconditioner is suitable. This is because $\boldsymbol{A q}=\boldsymbol{r}$ is solved simply in the preconditioning, and the computational time of the preconditioner is close to half of the entire computational time of the ICCG.

To parallelize the IC preconditioner, we used a multicoloring technique. In this study, we apply reverse Cuthill-Mckee and cyclic multicoloring (CM- RCM)[19]. The CM-RCM(nc) method has the advantage that the number of colors can be specified by users, and the effectiveness of the preconditioner is relatively good. In addition, by applying the CM-RCM(nc), as shown in Figure 3, the 
number of non-zero elements in each row of $\dot{L}$ and $\dot{D}$ is zero, three, or six.

\section{STORAGE FORMAT FOR SPARSE MATRIX}

In this section, we describe the storage format used in the evaluation.

Because the coefficient matrix of the SLEs derived from the FVM is sparse, the CRS storage format is generally used. However, it is not easy to use effective SIMDization with CRS. In this study, we also implement ELL and Sell-C- $\sigma$ storage formats that can use efficient SIMDization. Figure 4 shows difference among storage formats. In the ELL format, the elements of other lines are evenly padded with zeros to match the line with the largest number of non-zero elements. Therefore, non-zero elements can be stored in a twodimensional array, and it is easy to compile row-wise or columnwise SIMDization. Pseudo code 3 shows a forward substitution with color index $1 \sim n c$ in the IC preconditioner with the ELL storage format. In the pseudo-code, we expect the row-wise SIMDization. As described in section4.2, the number of non-zero elements per row is known and constant on each color. In the pseudo-code, the number of elements on each row is three. Therefore, there is no zero padding in the ELL, which is an ideal state.

The Sell-C- $\sigma$ format was proposed by Moritz Kreutzer et al. [9]. This storage format realizes fewer zero paddings, more effective SIMDization, and higher data locality compared with ELL. Sell-C$\sigma$ has two parameters: the sorting scope $\sigma$ and the chunk size $C$. Initially, the target matrix is divided into $\mathrm{N}$ uniform submatrices in the row direction, and the rows of the submatrix are sorted by the number of non-zero elements around the row. Then, the sorted submatrix is further divided into smaller submatrices with chunk size $C$ called "Chunk". In chunk, rows are zero-padded to match the length of the longest row. The chunk size $C$ is a parameter for SIMDization, and the optimal parameter $C$ is different among computer architectures. The parameter $\sigma$ determines that there is less zero paddings. It is better to select the parameter $\sigma$ that reduces

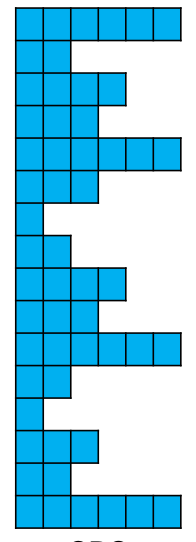

CRS

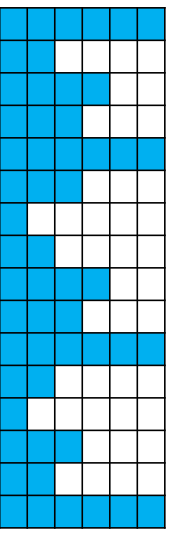

ELL

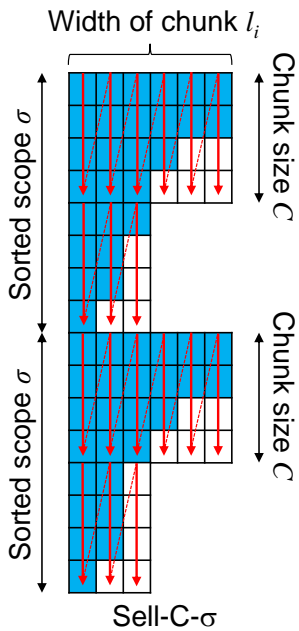

Figure 4: Difference among CRS, ELL and Sell-C- $\sigma$ storage format
Pseudo-code 3: Puseudo code of ELL

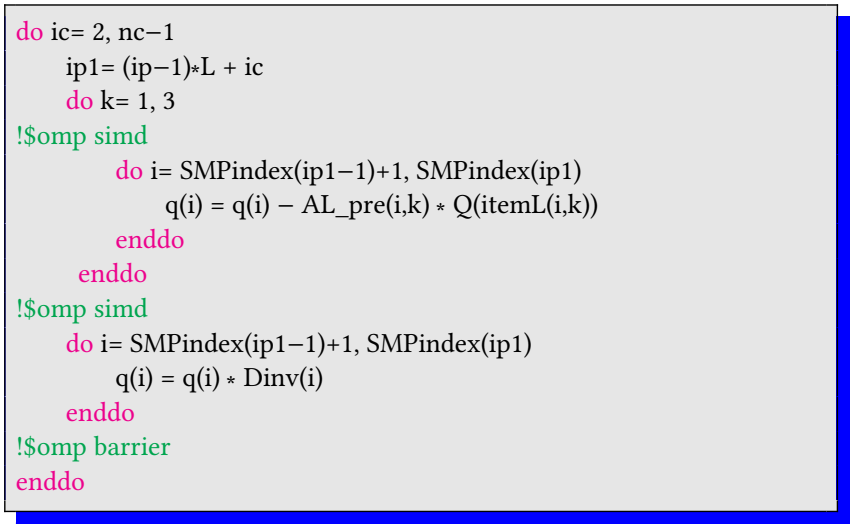

the number of zero paddings. Pseudo code4 shows the forward substitution with the Sell-C- $\sigma$ format. The "CHUNK" variable in the pseudo-code indicates the Chunk size $C$, and this parameter determines the SIMD length. Therefore, the parameter $C$ should be larger than the length of the SIMD instruction. Similar to the implementation of the ELL format, the number of non-zero elements in each row is constant and known. Therefore, we use the sorting scope $\sigma=1$ with no zero paddings, such as ELL. Then, we denote the original implementation(pseudo-code 4) as "base", and for optimizing the IC preconditioner with the Sell-C- $\sigma$ format, we have prepared other two implementations. The objective of the other optimized implementations is to increase the computations in the innermost loop for effective software pipelining. One is the moving "k" loop to the inside of the "is" loop, and it is denoted as "opt1". The other is unrolling the " $\mathrm{k}$ " loop of the "opt1" implementation, and it

\section{Pseudo-code 4: Puseudo code of Sell-C- $\sigma$}

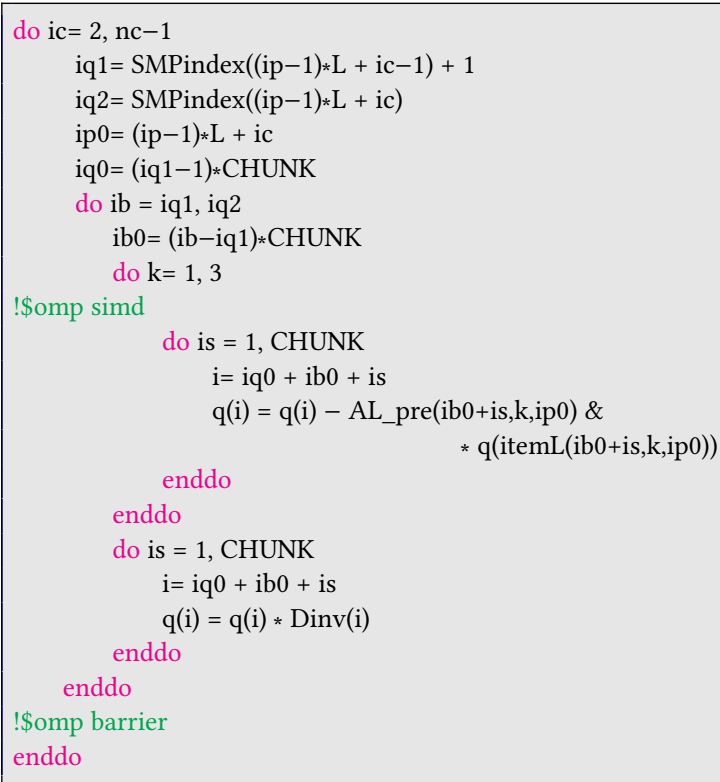


Table 2: System specifications

\begin{tabular}{|r|c|r|r|r|}
\hline \multicolumn{2}{|c|}{ Specifications } & Oakforest-PACS & Oakbridge-CX & Wisteria/BDEC-01 Odyssey \\
\hline \multirow{4}{*}{ CPU } & Model & $\begin{array}{r}\text { Xeon Phi 7250 } \\
\text { (Knights Landing) }\end{array}$ & $\begin{array}{r}\text { Xeon Platinum 8280 } \\
\text { (Cascade Lake) }\end{array}$ & A64FX \\
\cline { 2 - 5 } & Number of cores & 68 & 56 (2 Sockets) & $2.2 \mathrm{GHz}$ \\
\cline { 2 - 5 } & Clock & $1.4 \mathrm{GHz}$ & $2.7 \mathrm{GHz}$ & $64 \mathrm{kB} / \mathrm{core}$ \\
\cline { 2 - 5 } & L1-cache & $32 \mathrm{kB} / \mathrm{core}$ & $32 \mathrm{kB} / \mathrm{core}$ & $8 \mathrm{MB} / \mathrm{CMG}$ \\
\cline { 2 - 5 } & L2-cache & $1 \mathrm{MB} / 2 \mathrm{core}$ & $1 \mathrm{kB} / \mathrm{core}$ & $\mathrm{HBM} 2$ \\
\hline \multirow{3}{*}{ Memory } & Technology & MCDRAM & DDR4 & $32 \mathrm{~GB}$ \\
\cline { 2 - 5 } & Size & $16 \mathrm{~GB}$ & $192 \mathrm{~GB}$ & $840 \mathrm{~GB} / \mathrm{sec}$ \\
\cline { 2 - 5 } & Stream triad & $495 \mathrm{~GB} / \mathrm{sec}$ & $282 \mathrm{~GB} / \mathrm{sec}$ & \\
\hline
\end{tabular}

Table 3: Compiler and options on each system

\begin{tabular}{|c|c|r|l|}
\hline & Compiler & \multicolumn{1}{|c|}{ Version } & Options \\
\hline Oakforest-PACS & ifort & 19.1 .3 .304 & -O3 -xMIC-AVX512 -qopenmp -align array64byte -ipo \\
\hline Oakbridge-CX & ifort & 19.1 .3 .304 & -O3 -xHost -qqopenmp -align array64byte -ipo \\
\hline Wisteria/BDEC-01 Odyssey & frtpx & 4.5 .0 tcscd-1.2.31 & -O3 -Kfast,openmp,zfill,A64FX,ARMV8_A \\
\hline
\end{tabular}

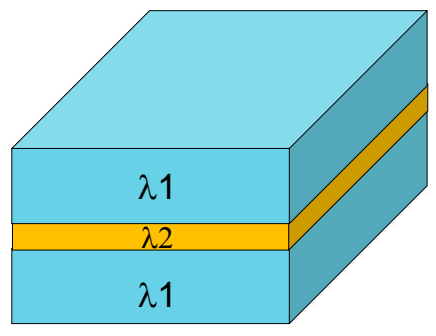

Figure 5: Distribution of the thermal diffusivity in the target problem

is denoted as "opt2". In the numerical experiment, we evaluated all storage formats such as CRS, ELL, Sell-C- $\sigma$ (base), Sell-C- $\sigma$ (opt1), and Sell-C- $\sigma$ (opt2).

The application of FP21 and FP42 requires load and storage for all three elements. Then, we only apply these adaptive precisions to the matrix and prepare two types of implementations. One is handling row-wise three elements for load and store, and the other is column-wise.

\section{NUMERICAL EVALUATIONS}

In this section, we show the result of numerical experiments. In the numerical experiments, we found the best implementation in a variety of storage formats and adaptive precision. By using the best implementation, we evaluate the effectiveness of the low- precision floating point for the condition of the problem.

\subsection{Environmens and conditions}

The systems used in the evaluations was Oakforest-PACS(OFP) operated by JCAHPC. We also used Oakbridge-CX(OBCX) and Wisteria/BDEC-01 Odyssey(WO) operated by the Information Technology Center at the University of Tokyo. Table 2 lists the specifications of and Table 3 shows the compiler options used for each system. Then, OFP and OBCX do not have hardware support for
FP16, so they are excluded from the evaluation of these systems. In addition, FP21 and FP42 are excluded from the evaluation of WO because of the limitations of the Fujitsu compiler.

The material of the target problem in this evaluation is a square shape with a length of one in all directions. The distribution of the thermal diffusivity is shown in figure 5 . In this study, we set the thermal diffusivity as $\lambda 1=1.0$ and $1.0 \leq \lambda \leq 10^{1} 0$. The target material is discretized with $n x, n y$, and $n z$ in each direction, and the number of degrees of freedom is $256^{3} \simeq 16.8$ million.

Regarding the ICCG method, all data types were implemented in FP64, excluding the IC preconditioner. The low-precision floatingpoint formats are only applied to the matrix and vector in the IC preconditioner. Different precisions can be applied to the matrices and vectors. The combinations of the floating-point formats of matrix and vector are denoted as "Matrix-vector" and the following were evaluated.
(1) FP64-FP64
(2) FP42-FP64
(3) FP32-FP64
(4) FP64-FP32
(5) FP32-FP32
(6) FP21-FP32
(7) FP16-FP32

The above order is in descending order of the amount of data transfer. It has been confirmed that if FP16 is applied to the vector, it does not converge. Therefore, the combination with FP16 vector is not included. The storage format of the matrix referred to in the matrix-vector multiplication in the CG method is the same as that of the IC preconditioner such as CRS, ELL, and Sell-C- $\sigma$. In the ICCG method, if the relative residual norm fulfills the condition $\left\|r^{k}\right\|_{2} /\left\|r^{0}\right\|_{2} \leq 10^{-8}$, the iteration is stopped because of judging as getting the approximate solution is obtained.

\subsection{Preliminary evaluation of storage formats}

In this section, we present the evaluation results for the performance of each storage format. The thermal diffusivity $\lambda$ is set to be 


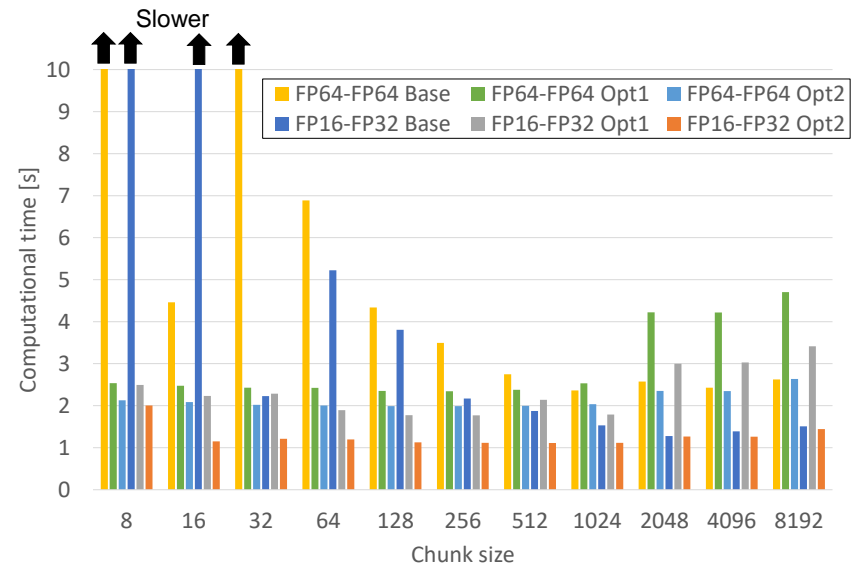

Figure 6: Comparison among implementations of Sell-C- $\sigma$ for Chunk size(FP16 matrix and FP32 vectors)

uniform in the object (the coefficient matrix is a well-conditioned). In this condition, the number of iterations on each floating-point format is equal, so the evaluation results show performance among storage formats.

The difference in performance because of the implementation of Sell-C- $\sigma$ is shown in Figure 6. This figure shows the computational time of the IC preconditioner on the WO system. As shown in Figure 6 , the "Opt2" implementation is the fastest in all Sell-C- $\sigma$ implementations, and the effect of applying the low- precision floating-point is high. To improve performance by applying low-precision floatingpoint formats, it is necessary to maintain sufficient memory access by using effective SIMDization. It is difficult to obtain effective SIMDization with "Base" implementation because of fewer computations in the most inner loop. In particular, when the chunk size is small, the "Base" implementation takes a long computational time compared to the others. Opt1 and Opt2 implementations are faster than the "Base" in any chunk size because of the sufficient amount of calculations. Especially for "Opt2" implementation, the software pipelining works effectively by loop unrolling, and it is the fastest. The same tendency was observed in the other systems, and "Opt2" was the fastest. Therefore, we show the result of "Opt2" in the subsequent evaluations.

Figure 7 shows the effect of changing the floating-point formats and chunk size on the computational time of the IC preconditioner. As shown in the figure, the optimum chunk size differs depending on the floating-point formats and systems. When we focus on the difference of floating-point formats, the larger chunk size is suitable to low-precision floating-point formats. It is because more operations are required for effective SIMDization with low-precision floating-point formats. On the result of OFP(Figure 7a), the optimum chunk size is as small as 8 to 16 , and it shows longer computational time with larger chunk size. On the OBCX system, the sensitivity to the chunk size is small compared with the other systems. The computational time with the optimum chunk size is reduced by about $1.3 \%$ compared to chunk size 8 . On the WO system, the effect of the difference in chunk size on the computational time is larger than the other systems. It was also found that a relatively large

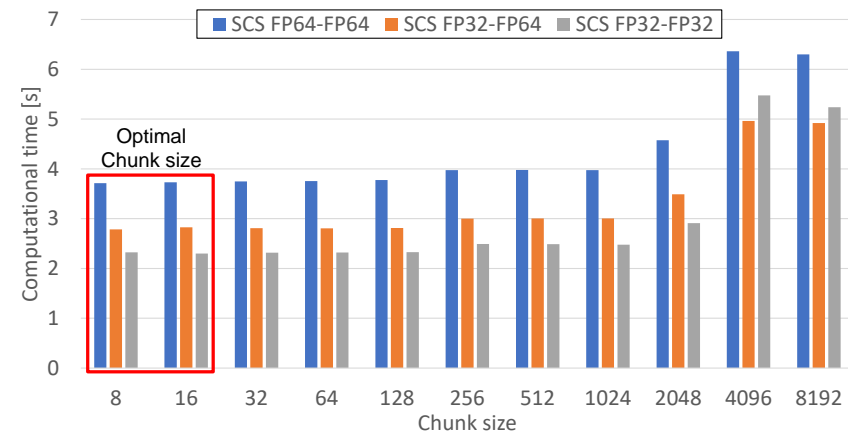

(a) Oakforest-PACS (XeonPhi)

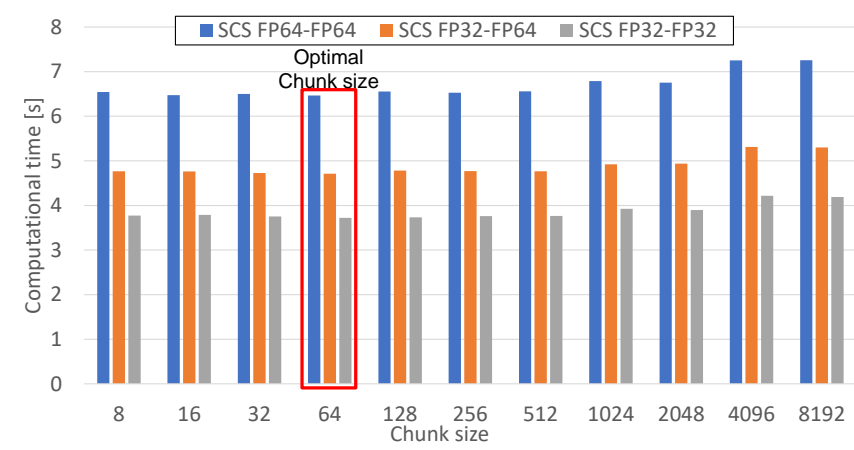

(b) Oakbridge-CX (CascadeLake)

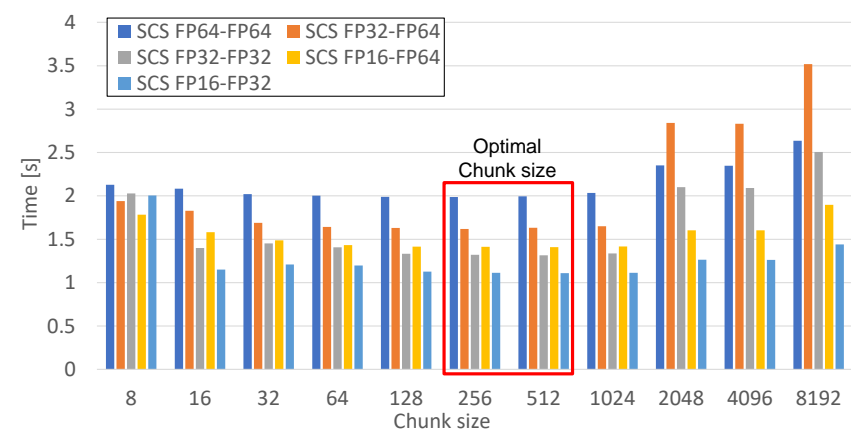

(c) Wisteria/BDEC-01 Odyssey (A64FX)

Figure 7: Computational time of each data format for Chunk size

Chunk size is optimum, and the chunk size has a large impact when using the low-precision. For example, the computational time of FP64-FP64 with a chunk size of 256 was $6.6 \%$ faster than that of chunk size 8 , and FP16-FP32 with chunk size 512 was $44.6 \%$ faster than the chunk size of 8 . In the comparison between FP16-FP64 and FP32-FP32, which have the same amount of memory access, FP16-FP64 was slower. This is because the calculation accuracy is converted to higher precision at the time of calculation, and a higher precision results in a smaller number of calculations per SIMD instruction. FP32-FP32 was calculated using FP32, and FP16FP64 was calculated using FP64. The number of calculations per SIMD instruction with FP16-FP64 was less than that of FP32-FP32. 


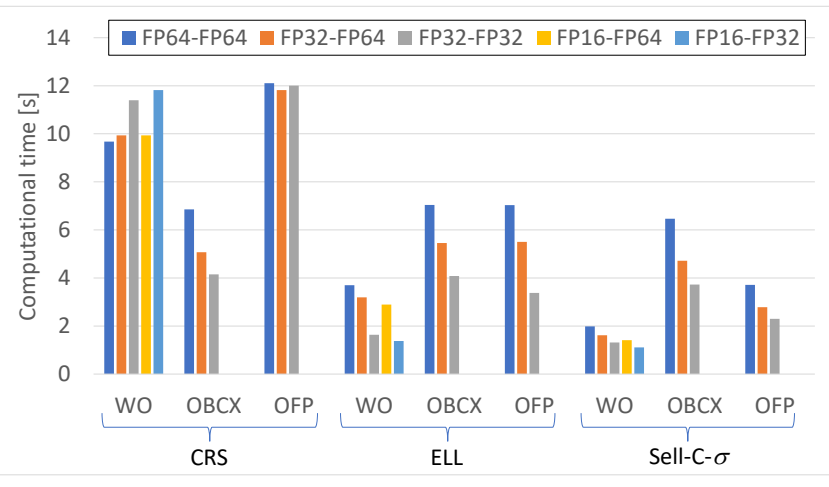

(a) Only IC preconditioner part

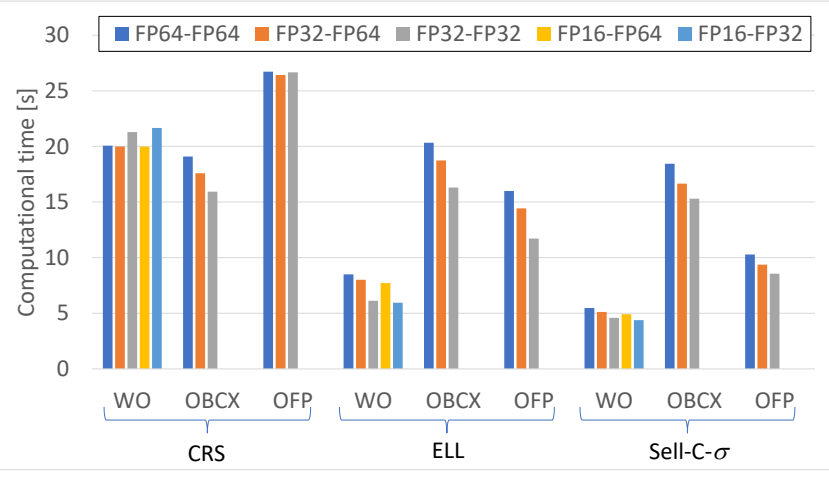

(b) Entire ICCG

Figure 8: Comparison among storage formats and systems

Figure 8a shows the calculation time of the IC preconditioner with different sparse matrix storage formats for each system. Figure $8 \mathrm{~b}$ shows the calculation time for the entire ICCG method. Then, the optimum chunk size is applied to Sell-C- $\sigma$ for each system. In the OBCX system, there was no large difference in any sparse matrix storage format, and the CRS format was the fastest of all systems. In OFP and WO, the effect of the Sell-C- $\sigma$ format is large. The Sell$\mathrm{C}-\sigma$ is faster for all storage formats on the OFP and WO. Sell-C- $\sigma$ with FP64-FP64 was $69.3 \%$, and FP32-FP32 was $80.8 \%$ faster than the CRS on OFP. In the WO system, Sell-C- $\sigma$ with FP64-FP64 was $79.5 \%$, and FP32-FP32 was $88.5 \%$ faster. Moreover, in the calculation time of the entire ICCG method, the difference in calculation time because of the difference in storage format is even more significant.

When we focus on the difference among precisions, a faster storage format shows a larger effect of low precision. The computational time of Sell-C- $\sigma$ with FP32-FP32 was $33.8 \%, 42.4 \%$, and $38.0 \%$ faster than that of FP64-FP64 on OFP, OBCX, and WO, respectively. FP16-FP32 was $44.1 \%$ faster than FP64-FP64 on WO. The effectiveness of the low-precision to the entire ICCG method is smaller than that of the IC preconditioner because of the limited scope of applying low precision. In subsequent evaluations, we used the Sell-C- $\sigma$ storage format.

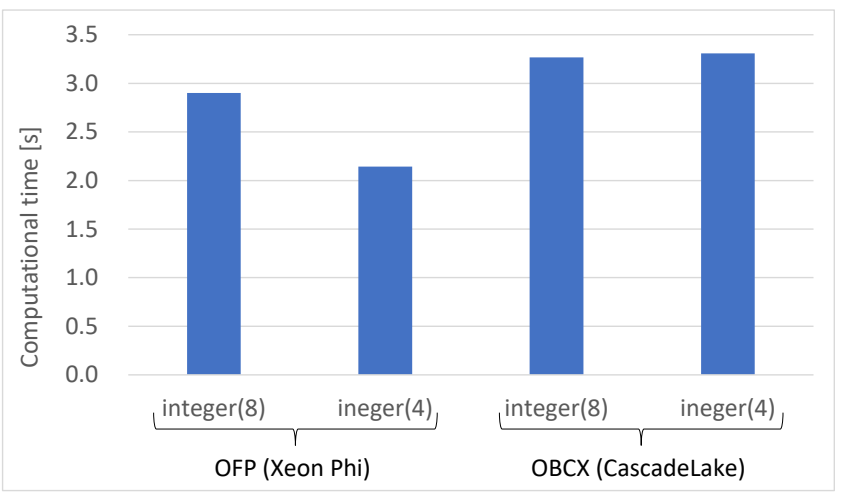

Figure 9: Comparison between storing data type of FP21

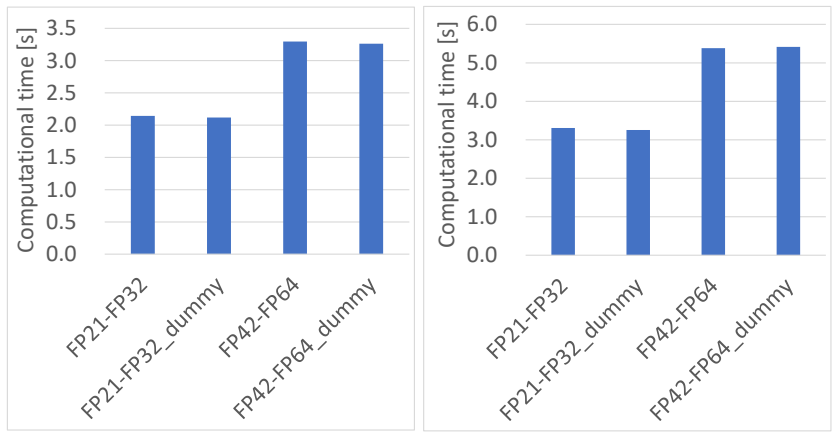

(a) Oakforest-PACS (XeonPhi) (b) Oakbridge-CX (CascadeLake)

\section{Figure 10: Overhead of loading FP21 and FP42}

\subsection{Preliminary evaluation of adaptive precision}

In this section, we perform a preliminary evaluation of adaptive precision.

Figure 9 shows the difference between 32-bits and 64- bits integer stored FP21 on the OFP and OBCX. On the OFP, the 32-bits integer is $26.1 \%$ faster than the 64-bits integer. As mentioned in Section 2.2, by using the 32-bits integer, the width of all reference data is equalized to 32-bit, and the number of computations per SIMD instruction becomes 16 with 512-bits SIMD. When we use the 64-bits integer, the number of computations per SIMD instruction becomes eight, despite referring data for computations as FP32.

Figure 10 shows the overhead of loading the FP21 and FP42 formats. To measure the overhead, we prepared a dummy code that changed the FP21 or FP42 loading function to normal loading with the same amount of reference data. The measuring overhead of adaptive precision was $1.2 \%$ in maximum for OFP and $1.5 \%$ for OBCX. We conclude that the overhead of loading an adaptive precision is sufficiently small.

Figure 11 shows the computational time of the IC preconditioner with row-wise and column-wise implementations of FP21-FP32 and FP42-FP64. On the OBCX, there is no difference between the rowwise and column-wise implementations. On the OFP, FP21-FP32 with column-wise implementation is close to three times faster 


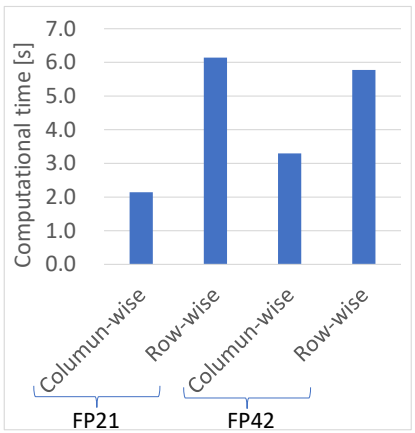

(a) Oakforest-PACS (XeonPhi)

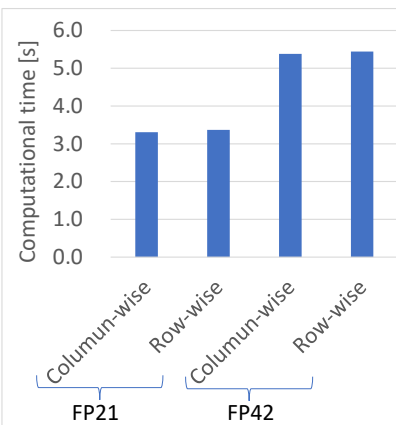

(b) Oakbridge-CX (CascadeLake)

Figure 11: Comparison of row-wise or column-wise implementation of FP21 and FP42

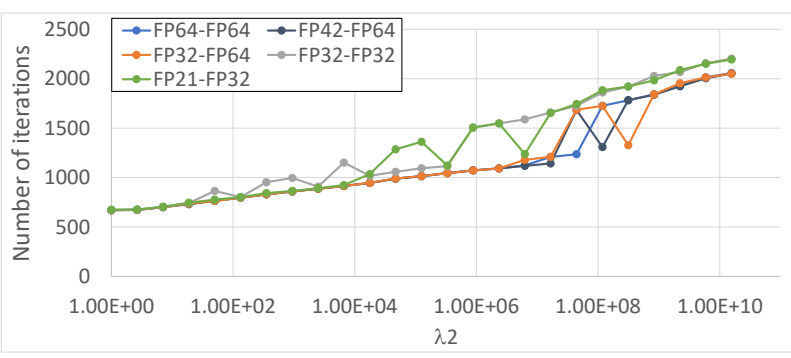

(a) Oakforest-PACS (XeonPhi)

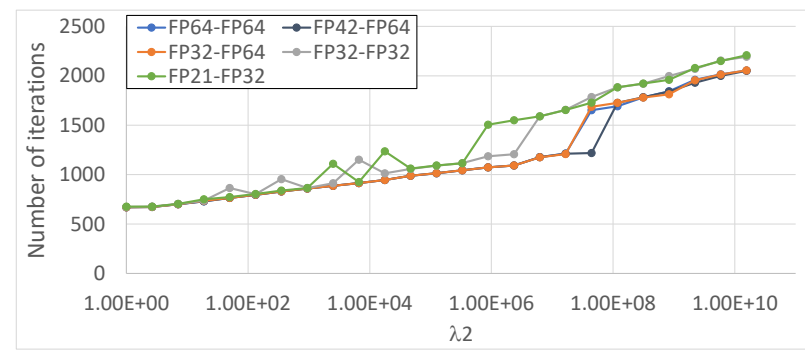

(b) Oakbridge-CX (CascadeLake)

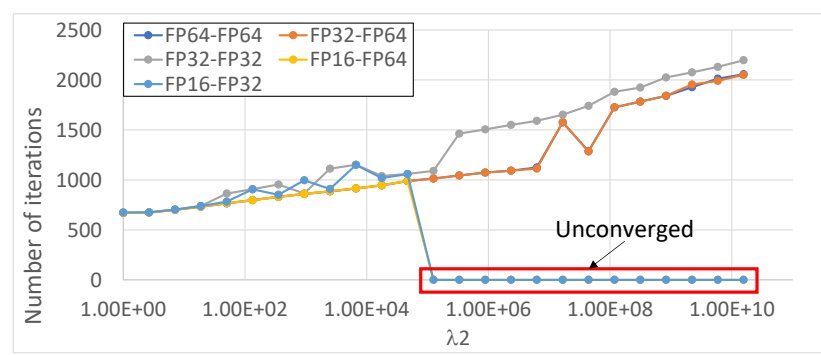

(c) Wisteria/BDEC-01 Odyssey (A64FX)

Figure 12: Effect of thermal difussivity $\lambda$ to convergence ratio

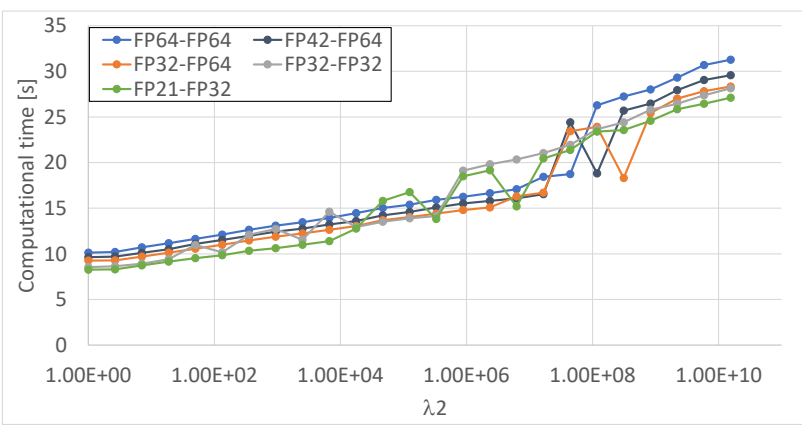

(a) Oakforest-PACS (XeonPhi)

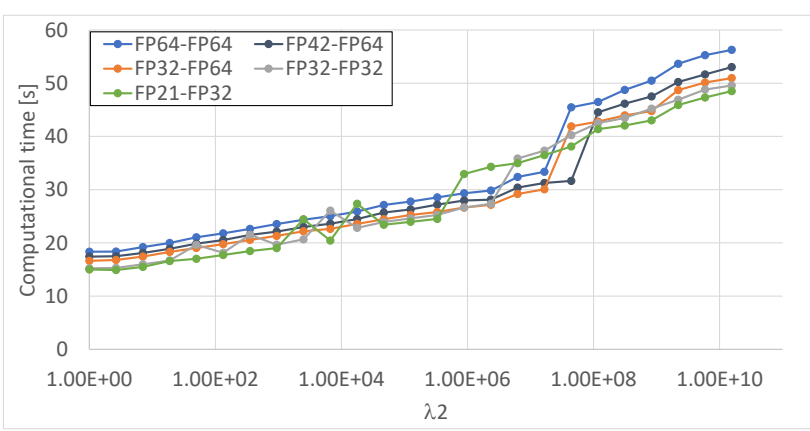

(b) Oakbridge-CX (CascadeLake)

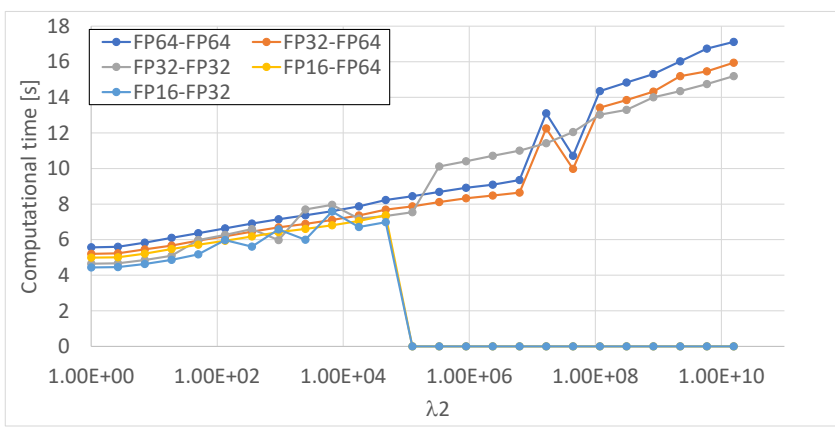

(c) Wisteria/BDEC-01 Odyssey (A64FX)

Figure 13: Effectiveness of low-precision to the computatinal for thermal difussivity $\lambda$

than the row-wise. In subsequent evaluations of adaptive precision, we use the column-wise implementation.

\subsection{Evaluation of low-precision}

In this section, to show the effect of low-precision floating-point formats, we evaluate the number of iterations and the computational time for each floating-point format when the thermal diffusivity $\lambda$ is changed. In the evaluation, we used the Sell-C- $\sigma$ formats, which was the fastest in all systems, and it has been confirmed in advance that the difference in storage format does not affect convergence.

Figure 12 and Figure 13 show the effect on convergence and computational, respectively. Using low- precision floating-point 
numbers on OFP, OBCX, and WO. As shown in Figure 12, the number of iterations shows a similar tendency for all the systems.

The slight differences are because of the compiler used and the degree of parallelism. As long as the coefficient matrix is in wellconditioned, there is no difference in convergence with any floatingpoint format. Under the condition $\lambda 2 / \lambda 1>20$, the convergence ratio using the FP32 vector is lower than that using the FP64 vectors. When $\lambda 2>10^{5}$, the FP16-FP32 IC preconditioner does not converge. This is because the non-zero elements of the coefficient matrix exceed the range of expression of FP16(Table 1). The ICCG method with the FP21 IC preconditioner converges under all conditions, and we indicate the importance of the expressive ability of the exponent part. From these results, if there is a factor that makes the condition number even slightly worse, it is necessary to carefully select the floating-point formats of the vector to consider the computational time and convergence ratio. It was also found that we can select only by whether its floating-point format can represent the value of the non-zero element.

In terms of calculation time, the difference in the tendency between the systems is also small. When $\lambda 2<20.0$, which is in well-conditioned, the active use of low precision reduces the calculation time. From $\lambda 2>20.0$, the fastest floating-point number changed depending on the conditions. We list the floating-point format combinations in the following order: FP16-FP32 (WO only), FP32-FP32, FP32-FP64, and FP32-FP32 with $\lambda 2=1.0$. In the condition $1.0<\lambda 2<20.0$, we reduced the calculation time with FP32-FP32 by $16.8 \%$ on average on OFP and OBCX, and $16.5 \%$ on WO compared with FP64-FP64. We also archive to reduce the calculation time with FP16-FP32 by $20.3 \%$.

The use of FP21 contributed to the reduction in calculation time, and the calculation time was reduced by $11.0 \%$ in the evaluation of only the IC preconditioner with FP21-FP32 and by $3.0 \%$ in the entire ICCG method. In addition, the calculation time of FP42-FP64 was reduced by $13.6 \%$ only in the IC preprocessing section and by $5.4 \%$ in the entire ICCG method. In the ill-conditioned condition, FP32-FP32 was the fastest. This is because the convergence of the FP64 vector deteriorates, and it approaches the FP32 vectors.

\section{CONCLUSION}

In this study, we examined the effectiveness of low-precision and adaptive precision floating-point formats on the application. In the adaptive precision, we use the FP21 and FP42. FP21 was proposed for and evaluated on GPUs. In contrast, we optimized it for CPUs. For the application for the examination, we chose the ICCG method for solving the systems of linear equations(SLEs) derived from P3D. In P3D, the condition of the coefficient matrix of the SLE can be easily changed by controlling the thermal diffusivity. In addition, P3D is implemented with CRS, ELL and Sell-C- $\sigma$ as the storage format for the sparse matrix. It is important to maintain the pressure on the memory accesses to decrease the computational time by using low precision. Therefore, effective SIMDization is required in useful storage formats such as ELL and Sell-C- $\sigma$. In the numerical evaluation of the storage formats, Sell-C- $\sigma$ is the fastest, and we used Sell-C- $\sigma$ for the other numerical evaluations. In the existing research of FP21, it is stored as a 64-bits integer data type. In contrast, we propose storing a 32-bits integer. Using our approach, the performance with FP21 increased by $26.1 \%$. We also propose the FP42 format. In the numerical evaluations, we confirmed the effectiveness of the low- precision and adaptive precisions. To evaluate the effectiveness of low-precision and adaptive precision floating-point formats, we applied them to the IC preconditioner. With the wellconditioned of the coefficient matrix, the number of iterations is the same among all precision floating-point formats. Then, the combination of low-precision, such as the FP16 coefficient matrix and FP32 vector, is the fastest. In fact, the FP16 matrix and FP32 vector combination are $20.3 \%$ faster than the FP64 matrix and vector. With the ill-condition, the number of iterations is changed using precision, and the fastest combination is also changed depending on the number of iterations. When we use the FP16 coefficient matirx, it was not converged because of exceeding the expressive of exponent part. Therefore, when developing practical applications or libraries with the low-precisions, it is necessary to select the floating-point format considering a maximum of minimum non-zero element in the coefficient matrix.

In future research, we will examine auto-tuning so that the optimum precision can be selected automatically. We also apply low precision to the entire ICCG method. In this situation, we must decrease the accuracy of the approximate solution and convergence ratio.

\section{REFERENCES}

[1] [n.d.]. TensorFlow bfloat. https://github.com/tensorflow/tensorflow/blob/master/ tensorflow/core/framework/bfloat16.h.

[2] 2008. IEEE Standard for Floating-Point Arithmetic. IEEE Std 754-2008 (2008), $1-70$.

[3] Nathan Bell and Michael Garland. 2009. Implementing sparse matrix-vector multiplication on throughput-oriented processors. In Proceedings of the conference on high performance computing networking, storage and analysis. 1-11.

[4] M.A. Clark, R. Babich, K. Barros, R.C. Brower, and C. Rebbi. 2010. Solving lattice QCD systems of equations using mixed precision solvers on GPUs. Computer Physics Communications 181, 9 (2010), 1517 - 1528.

[5] Kohei Fujita, Masashi Horikoshi, Tsuyoshi Ichimura, Larry Meadows, Kengo Nakajima, Muneo Hori, and Lalith Maddegedara. 2020. Development of elementby-element kernel algorithms in unstructured finite-element solvers for manycore wide-SIMD CPUs: Application to earthquake simulation. fournal of Computational Science 45 (2020), 101174.

[6] A. Haidar, S. Tomov, J. Dongarra, and N. J. Higham. 2018. Harnessing GPU Tensor Cores for Fast FP16 Arithmetic to Speed up Mixed-Precision Iterative Refinement Solvers. In SC18: International Conference for High Performance Computing, Networking, Storage and Analysis. 603-613.

[7] Berk Hess. 2008. P-LINCS : A Parallel Linear Constraint Solver for Molecular Simulation. Fournal of Chemical Theory and Computation 4, 1 (2008), 116-122.

[8] Wei Jiang, Ziwei Song, Jinyu Zhan, Zhiyuan He, Xiangyu Wen, and Ke Jiang. 2020. Optimized co-scheduling of mixed-precision neural network accelerator for real-time multitasking applications. Journal of Systems Architecture 110 (2020), 101775.

[9] Moritz Kreutzer, Georg Hager, Gerhard Wellein, Holger Fehske, and Alan R Bishop. 2014. A unified sparse matrix data format for efficient general sparse matrix-vector multiplication on modern processors with wide SIMD units. SIAM fournal on Scientific Computing 36, 5 (2014), C401-C423.

[10] Scott Le Grand, Andreas W. Götz, and Ross C. Walker. 2013. SPFP: Speed without compromise-A mixed precision model for GPU accelerated molecular dynamics simulations. Computer Physics Communications 184, 2 (2013), $374-380$.

[11] J. Lee, J. Lee, D. Han, J. Lee, G. Park, and H. Yoo. 2019. 7.7 LNPU: A 25.3TFLOPS/W Sparse Deep-Neural-Network Learning Processor with Fine-Grained Mixed Precision of FP8-FP16. In 2019 IEEE International Solid-State Circuits Conference (ISSCC). 142-144.

[12] Naoto Matsuoka, Luyan Qiu, Xinyi Li, Tatsuya Omori, and Ken ya Hashimoto. 2019. Applicability of single precision graphics processing unit for fast simulation of $2 \mathrm{D}$ surface acoustic wave devices using an hierarchical cascading technique. fapanese fournal of Applied Physics 58, SG (jun 2019), SGGC11.

[13] S. R. Nandakumar, Manuel Le Gallo, Christophe Piveteau, Vinay Joshi, Giovanni Mariani, Irem Boybat, Geethan Karunaratne, Riduan Khaddam-Aljameh, Urs 
Egger, Anastasios Petropoulos, Theodore Antonakopoulos, Bipin Rajendran, Abu Sebastian, and Evangelos Eleftheriou. 2020. Mixed-Precision Deep Learning Based on Computational Memory. Frontiers in Neuroscience 14 (2020), 406.

[14] Kyaw L Oo and Andreas Vogel. 2020. Accelerating geometric multigrid preconditioning with half-precision arithmetic on GPUs. arXiv preprint arXiv:2007.07539 (2020).

[15] Rise Ooi, Takeshi Iwashita, Takeshi Fukaya, Akihiro Ida, and Rio Yokota. 2020 Effect of Mixed Precision Computing on H-Matrix Vector Multiplication in BEM Analysis. In Proceedings of the International Conference on High Performance Computing in Asia-Pacific Region (Fukuoka, Japan) (HPCAsia2020). Association for Computing Machinery, New York, NY, USA, 92-101.

[16] Ryuichi Sakamoto, Masaaki Kondo, Kohei Fujita, Tsuyoshi Ichimura, and Kengo Nakajima. 2020. The Effectiveness of Low-Precision Floating Arithmetic on Numerical Codes: A Case Study on Power Consumption (HPCAsia2020). 199-206.

[17] Ryuichi Sakamoto, Masaaki Kondo, Kohei Fujita, Tsuyoshi Ichimura, and Kengo Nakajima. 2020. The Effectiveness of Low-Precision Floating Arithmetic on
Numerical Codes: A Case Study on Power Consumption. In Proceedings of the International Conference on High Performance Computing in Asia-Pacific Region (Fukuoka, Japan) (HPCAsia2020). Association for Computing Machinery, New York, NY, USA, 199-206.

[18] A. Walden, E. Nielsen, B. Diskin, and M. Zubair. 2019. A Mixed Precision Multicolor Point-Implicit Solver for Unstructured Grids on GPUs. In 2019 IEEE/ACM 9th Workshop on Irregular Applications: Architectures and Algorithms (IA3). 23-30.

[19] T Washio, K Maruyama, T Osoda, F Shimizu, et al. 2000. Efficient implementations of block sparse matrix operations on shared memory vector machines. In SNA 2000. The fourth international conference on supercomputing in nuclear applications.

[20] Takuma Yamaguchi, Kohei Fujita, Tsuyoshi Ichimura, Akira Naruse, Maddegedara Lalith, and Muneo Hori. 2019. GPU implementation of a sophisticated implicit low-order finite element solver with FP21-32-64 computation using OpenACC. Lecture Notes in Computer Science, WACCPD 201912017 (2019). 